\title{
Intestinal obstruction in an elderly man
}

\author{
K Azimuddin, R Bourne
}

A 75-year-old man was admitted with a two-day history of colicky abdominal pain, vomiting and absolute constipation. On examination he looked unwell, dysponeic, and dehydrated. The abdomen was distended, tympanic and generally tender. Bowel sounds were obstructive. Routine blood tests were normal except for a marginally raised white cell count. The plain abdominal $\mathrm{X}$-ray is shown in figure 1 .

Department of General Surgery, North Devon Hospital, Barnstaple, Devon, EX31 4JB, UK

K Azimuddin

R Bourne

Correspondence to

Mr R Bourne

Accepted 3 May 1995

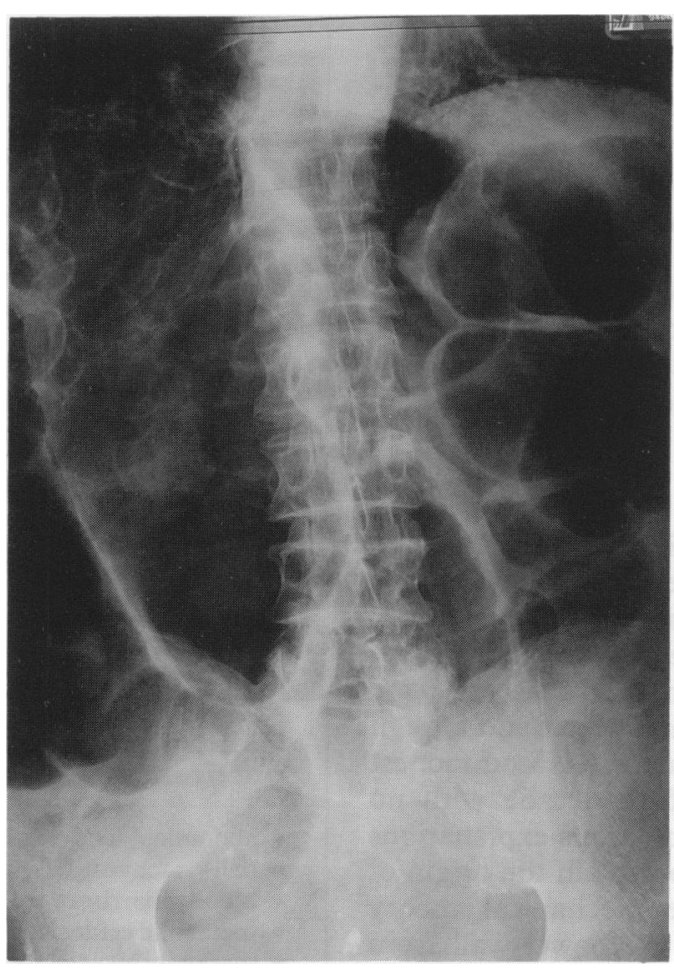

\section{Questions}

1 What are the two important features seen in the abdominal X-ray?

2 What is the diagnosis?

3 What is the immediate management of this condition?

Figure 1 Plain abdominal X-ray 


\section{Answers}

QUESTION 1

There is a gigantic distension of large bowel on the right side consistent with volvulus of the sigmoid colon. Secondly, numerous round, translucent cysts are seen within the wall of the twisted loop.

\section{QUESTION 2}

The diagnosis is intestinal obstruction secondary to volvulus of the sigmoid colon. The appearance of pneumatosis suggests that the ischaemic bowel is no longer viable.

\section{QUESTION 3}

The patient should be immediately resuscitated with intravenous fluids. A central venous line should be placed to help with fluid replacement. Pre-operative antibiotics may be required depending upon the degree of systemic sepsis.

An urgent laparotomy is required. A Hartman's procedure should be performed with resection of the affected segment of bowel. By the time pneumatosis has appeared in this situation there is already a degree of mucosal ischaemia and necrosis/performation is imminent. Therefore colonoscopic decompression, untwisting followed by sigmoidoplexy or simple loop colostomy should not be undertaken.

\section{Discussion}

Pneumatosis cystoides intestinalis (PCI) is characterised by the presence of multiple cysts within the bowel wall and/or its mesentery (figure 2). The cysts range from few millimetres to several centimetres in size and some are lined, often partially, by histiocytes and foreign body giant cells. Pneumatosis is commonest during the fourth and fifth decade with no sexual predominance. Numerous explanations have been put forward to explain the origin of air in these cysts. The mechanical theory suggests that gas enters the bowel wall from either its luminal or serosal aspect, whereas according to the bacterial theory cysts are produced as a result of the metabolic activity of gas-forming organisms. Box 1 shows a list of conditions in which pneumatosis is encountered. The association of pneumatosis

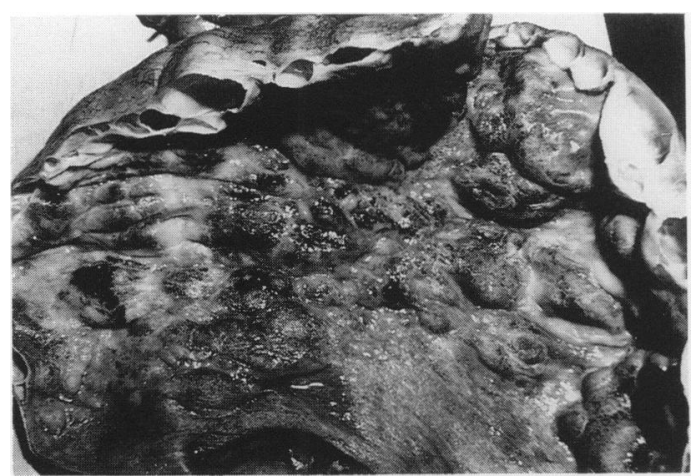

Figure 2 Multiple bowel wall cysts characteristic of pneumatosis cystoides intestinalis
Current theories on origin of pneumatosis

Gas entering bowel wall from mucosal aspect

Following mucosal breach:

- ulcers - benign (peptic) or malignant

- infections - viral, bacterial or tuberculous

- inflammatory bowel disease - Crohns, ulcerative colitis

- ischaemia - ischaemic colitis, mesenteric infarct or embolus, volvulus, strangulation, narcotising enterocolitis

- iatrogenic - endoscopy and polypectomy, barium enema, placement of enteric tubes, intestinal anastamosis

- vasculitis - connective tissue disorders (systemic sclerosis, dermatomyositis), graft vs host disease, radiation enteritis

Due to high intraluminal pressure:

- mechanical bowel obstruction - bowel tumours, benign strictures, obstructed hernia, volvulus, idiopathic megacolon, pyloric stenosis from peptic ulcers

- dynamic obstruction - pseudo-obstruction, chronic intestinal distension

Gas-forming bacteria entering bowel wall

- eg, Clostrida, from any of the above

- lowered mucosal resistance immunosuppression (steroid therapy, leukaemia, transplantation), chemotherapy, whole body irradiation

Gas entering bowel wall from serosal aspect

- after dissecting through interstitium of lungs, mediastinum and retroperitoneum - chronic obstructive airway disease, cystic fibrosis, bronchopulmonary dysplasia, positive pressure ventilation

Box 1

Indications for early laparotomy in pneumatosis

- abdominal tenderness, guarding, rigidity

- fever, tachycardia, hypotension

- bloody diarrhoea

- metabolic acidosis

- markedly elevated serum amylase

Box 2

with so many varied conditions suggests that PCI is a sign rather than a disease in itself.

Clinically, PCI usually runs an asymptomatic course and is often incidentally picked up on radiographic examination. Occasionally, multiple cysts in the bowel lumen can cause obstruction and pain. The commonest presenting symptom in the benign form of PCI is nausea, vomiting, diarrhoea and discharge of mucous and blood per rectum, the bleeding usually coming from congested mucosa overlying the cysts. Rarely, the cysts rupture, leading to pneumoperitoneum without peritonitis. The priority then is to differentiate the condition from surgical causes of pneumoperitoneum and thereby avoid unnecessary laparotomy. It is only when pneumatosis results from bowel ischaemia, that it presents a fulminating course. 
The underlying aetiology of PCI, ie, mesenteric ischaemia or bowel infarction, is then the presenting disease. The natural course of PCI, except when it is secondary to bowel ischaemia, is usually benign. It has been suggested that gross pneumatosis and pneumatosis lasting longer than a few days also implies a benign course. Many cysts disappear spontaneously and may never come to notice.

The diagnosis of PCI is usually made on a plain X-ray of the abdomen, on which it appears as translucent clusters along the margins of the bowel wall. On endoscopy the sub-mucosal cystic lesions project into lumen like multiple polyps, which tend to bleed on contact. Barium enema shows multiple rounded filling defects and the picture can be mistaken for polyposis, carcinoma or ulcerative colitis. Ultrasound shows a thickened bowel wall with multiple cystic lesions and is more sensitive than a plain radiograph. Computed tomography is most sensitive but not used for routine clinical evaluation.

1 Knechtle SJ, Davidoff AM, Rice RP. Pneumatosis intestinalis, surgical management and clinical outcome. Ann Surg 1990; 12: 160-5.

2 Rogy MA, Mirza DF, Kovats E, Rauhs R. Pneumatosis cystoides intestinalis. Int $\mathcal{f}$ Colorectal Dis 1990; 5: 120-4.

3 Luks FI, Chung MA, Brandt ML, Hertecant J, Roy CC Blanchard H. Pneumatosis and pneumoperitoneum in chronic idiopathic intestinal pseudo-obstruction. $\mathcal{F}$ Pediat Surg 1991; 26: 1384-6.

4 Kelly B, Cooper GG, Mills J. Pneumoperitoneum due to $\mathrm{PCI}$ in a patient with strangulated femoral hernia. $\mathrm{Br} \mathcal{f} \mathrm{Clin}$ Pract 1993; 47: 272 .

5 Yale CE, Balish E. The natural course of Clostridium perfringens-induced pneumatosis cystoides intestinalis. $\mathscr{F}$ Med 1992; 23: 279-88.
The treatment of pneumatosis secondary to ischaemia requires prompt resection of the affected bowel. Box 2 enumerates factors which suggest bowel ischaemia or gangrene in a case of pneumatosis. These patients have a poor prognosis and should undergo urgent laparotomy. In its 'benign' non-ischaemic form, PCI rarely requires treatment unless symptomatic in which case metronidazole, amoxycillin or hyperbaric oxygen can be used. However, as the 'benign' form is often self-limiting it is doubtful whether any treatment is required at all.

\section{Final diagnosis}

Pneumatosis cystoides intestinalis

Keywords: pneumatosis cystoides intestinalis, sigmoid volvulus
Acute Elderly Services, Orsett Hospital, Orsett, Grays, Essex RM16 3EU, UK GH Jenner

Accepted 1 June 1995
6 Van Leeuwen JC, Nossent JC. Pneumatosis intestinalis in mixed connective tissue disease. Neth $\mathcal{F}$ Med 1992; 40 299-302.

7 Jensen R, Gutnik SH. Pneumatosis cystoides intestinalis, a complication of colonscopic polypectomy. $S$ D $\mathcal{Y}$ Med 1991; 44: 177-9.

8 Cho KC, Simmons MZ, Baker SR, Cappell MS. Spontaneous dissection of air into the transverse mesocolon during double-contrast barium enema. Gastrointest Radio 1990; 15: 76-7.

9 Frimann-Dahl J. Roentgen examination in acute abdominal disease. 3rd edn, 1974; $\mathrm{pp}$ 55-9.

10 Williams JL. Pneumatosis cystoides intestinalis involving the left half of the colon and rectum. Br $\mathcal{F}$ Surg 1961; 49: 67-9.

\title{
A seizure and odd behaviour in a 79-year-old man
}

\author{
GH Jenner
}

A 79-year-old man, widowed one week, presented to the Accident and Emergency department unconscious following a grand mal epileptic fit. His daughter described episodes of peculiar behaviour over the last six months, including one lasting 30 minutes when he woke from sleep shouting "I must save Mr Gorbachev". Laboratory results were as follows: urea: $8.0 \mathrm{~mm} / 1$ (normal <6.7 mm/1); electrolytes: sodium $141 \mathrm{~mm} / 1(135-145 \mathrm{~mm} / 1$ ), potassium $4.7 \mathrm{~mm} / 1$ (3.5-5.0 mm/1); blood sugar $<1 \mathrm{~mm} / 1$. Electrocardiogram showed left bundle branch block.

He was admitted to hospital. Two and a half hours later he became violent and abusive. A bedside finger prick blood glucose BM Stix was $<1.7 \mathrm{~mm} / 1$.

\section{Questions}

1 What is the most likely cause of his presenting symptoms?

2 What diagnoses should be considered and why?

3 What investigations would be helpful?

4 What are the options for treatment in the short and long term? 